\title{
Cadena en bloques y la trazabilidad en la cadena de suministro agroalimentaria
}

\author{
Blockchain and traceability in the agri-food supply chain \\ Rafael Granillo-Macias ${ }^{a}$
}

\begin{abstract}
:
The advantage of using Blockchain traceability solution is the availability of verifying the information by all the parts that make up a supply chain. The records that are made in Blockchain are immutable, ensuring the originality and authenticity of the information, this characteristic towards traceability makes this tool a competitive advantage for the agri-food sector.
\end{abstract}

Keywords:

Industry 4.0, smart agriculture, blockchain

\section{Resumen:}

La ventaja que representa la utilización de una solución para la trazabilidad mediante cadena en bloques, es la disponibilidad de verificar la información por todas las partes que conforman una cadena de suministro. Los registros que se realizan en la cadena de bloques son inmutables, asegurando la originalidad y autenticidad de la información, esta característica hacia la trazabilidad hace de esta herramienta una ventaja competitiva para el sector agroalimentario.

Palabras Clave:

Industria 4.0, agricultura inteligente, cadena en bloques

\section{Introducción}

Según estimaciones de la Organización de las Naciones Unidades para la Agricultura y la Alimentación (FAO, por sus siglas en Ingles), el sector agroalimentario tendrá que aumentar sus rendimientos de manera sostenible en un $70 \%$ para el 2050. Ante este escenario de creciente demanda de alimentos, y que podría derivar en una crisis alimentaria, la necesidad de una "agricultura inteligente" es inminente y necesaria para asegurar la sostenibilidad del propio sector y de las cadenas de suministros que se interrelacionan con este.

\section{Descripción}

El concepto de la tecnología de Cadena en Bloques se define como una base de datos distribuida de registros en forma de "bloques" (pequeña base de datos) encriptados que contienen todas las transacciones o eventos digitales que se han ejecutado y compartido entre las partes participantes y se pueden verificar en cualquier momento en el futuro (Antonucci et al., 2019). Las transacciones son verificadas por consenso de la mayoría de los participantes en el sistema, mediante un protocolo de comunicaciones estándar y que es accesible a todos ellos, eliminando la necesidad de acuses de recibo y conciliaciones (Borrero, 2019).

\footnotetext{
a Universidad Autónoma del Estado de Hidalgo, Escuela Superior de Ciudad Sahagún, https://orcid.org/0000-0002-1015-667X, Email: rafaelgm@uaeh.edu.mx
} 
La trazabilidad mediante la cadena en bloques, tiene como objetivo el registrar toda la información relacionada con la cadena de suministro desde las operaciones con proveedores hasta a distribución de los productos, además de identificar y asociar datos importantes sobre las condiciones ambientales y métodos de producción. El enfoque adoptado para la cadena de suministro agroalimentaria se basa en una perspectiva de múltiples actores, integrando herramientas para la participación en la toma de decisiones de forma consensada.

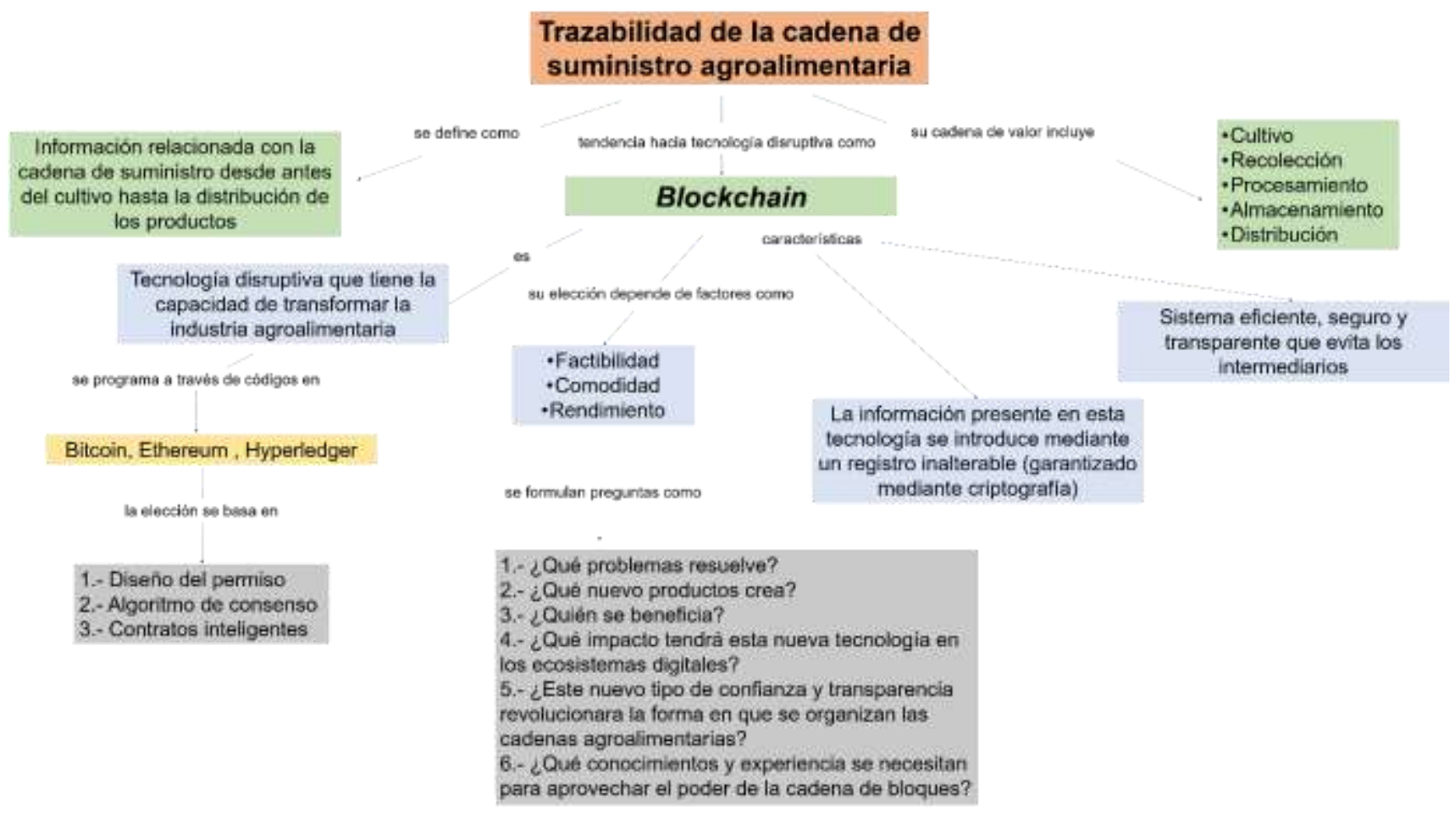

Figura 1. Cadena en bloques y la trazabilidad en la cadena de suministro agroalimentaria

\section{Referencias}

Antonucci, F., Figorilli, S., Costa, C., Pallottino, F., Raso, L., \& Menesatti, P. (2019). A review on blockchain applications in the agri-food sector. Journal of the Science of Food and Agriculture, 99(14), 6129-6138. https://doi.org/10.1002/jsfa.9912

Borrero, J.D. (2019). Sistema de trazabilidad de la cadena de suministro agroalimentario para cooperativas de frutas y hortalizas basado en la tecnología Blockchain. CIREC. Revista de Economía Publica, Social y Cooperativa. 95, pp. 71-94.

FAO. (2020). Food and Agriculture Organization of the United Nations FAOSTAT. http://www.fao.org/faostat/es/\#data/QC 